\title{
Correlation of electronic carotenoid-chlorophyll interactions and fluorescence quenching with the aggregation of native LHC II and chlorophyll deficient mutants
}

\author{
Pen-Nan Liao ${ }^{\mathrm{a}}$, Stefan Bode ${ }^{\mathrm{a}}$, Laura Wilk ${ }^{\mathrm{b}}$, Nour Hafi ${ }^{\mathrm{a}}$, Peter J. Walla ${ }^{\mathrm{a}, \mathrm{c}, *}$ \\ ${ }^{a}$ Technische Universität Braunschweig, Institute for Physical and Theoretical Chemistry, Department for Biophysical Chemistry, Hans-Sommer-Strasse 10, 38106 \\ Braunschweig, Germany \\ ${ }^{\mathrm{b}}$ Max Planck Institute of Biophysics, Department of Structural Biology, Max-von-Laue-Strasse 3, 60438 Frankfurt am Main, Germany \\ ${ }^{\mathrm{c}}$ Max Planck Institute for Biophysical Chemistry, Department of Spectroscopy and Photochemical Kinetics, Am Fassberg 11, 37077 Göttingen, Germany
}

\section{A R T I C L E I N F O}

\section{Article history:}

Received 30 September 2009

In final form 14 January 2010

Available online 21 January 2010

\section{Keywords:}

LHC II

Two-photon excitation

Photosynthetic regulation

Carotenoids

Chlorophylls

Aggregation

\begin{abstract}
A B S T R A C T
The aggregation dependent correlation between fluorescence quenching and the electronic carotenoidchlorophyll interactions, $\phi_{\text {Coupling }}^{\mathrm{Car} \mathrm{S}_{1}-\mathrm{Chl}}$, as measured by comparing chlorophyll fluorescence observed after two- and one-photon excitation, has been investigated using native LHC II samples as well as mutants lacking Chl 2 and Chl 13. For native LHC II the same linear correlation between $\phi_{\text {Coupling }}^{\text {Car } s_{1}-\mathrm{Chl}}$ and the fluorescence quenching was observed as previously reported for the $\mathrm{pH}$ and Zea-dependent quenching of LHC II [1]. In order to elucidate which carotenoid-chlorophyll pair might dominate this correlation we also investigated the mutants lacking $\mathrm{Chl} 2$ and $\mathrm{Chl} 13$. However, also with these mutants the same linear correlation as for native LHC II was observed. This provides indication that these two chlorophylls play only a minor role for the observed effects. Nevertheless, we also conclude that this does not exclude that their neighboured carotenoids, lutein 1 and neoxanthin, might interact electronically with other chlorophylls close by.
\end{abstract}

(c) 2010 Elsevier B.V. All rights reserved.

\section{Introduction}

In a typical day plants are exposed to sudden changes of light intensities which could vary over several orders of magnitude [2]. Under low light conditions nearly all absorbed sun light quanta are transferred to the reaction centers of photosystems (PS) II and I where the energy conversion of electronic excitation energy into biochemical energy starts. However, under saturating high light conditions a protective mechanism often called non-photochemical quenching (NPQ) has to be initiated to prevent the photosynthetic apparatus from photo oxidative damage caused by excess energy. Balancing with high efficiency between energy utilisation and energy dissipation is essential for the survival and fitness of plants [3]. Non-photochemical quenching is a process which can dissipate solar energy which exceeds the photosynthetic capacity as harmless heat, minimizing harmful effects of photosynthetic by-products. In higher plants the major component of non-photochemical quenching is energy-dependent quenching ( $\mathrm{qE}$ ) [4,5]. qE is a fast reversible process which occurs on a time scale of seconds to minutes and depends on the trans-thylakoid $\mathrm{pH}$ gradient $(\Delta \mathrm{pH})$

\footnotetext{
* Corresponding author. Address: Technische Universität Braunschweig, Institute for Physical and Theoretical Chemistry, Department for Biophysical Chemistry, Hans-Sommer-Strasse 10, 38106 Braunschweig, Germany. Tel.: +49 5512011087; fax: +495313915352.

E-mail address: pwalla@gwdg.de (P.J. Walla).
}

$[4,6]$, the presence of the protein PsbS [7] as well as the xanthophyll cycle carotenoid zeaxanthin (Zea) [5]. In recent years many attempts have been performed in order to understand the physical mechanism of this important regulation and several models have been proposed for its explanation. However, at present even the exact site of this regulation within the photosynthetic apparatus is still a matter of intensive debate [7-14].

Light harvesting complex II (LHC II) is the major pigment-protein complex of higher plants, which is located at the periphery of PS II. The main function of LHC II is to collect the solar energy and transfer it to the reaction centers. Several studies suggest that LHC II plays a key role in the regulation of $\mathrm{qE}$ by switching into a quenched state under high light conditions due to mechanisms that are so far not completely understood $[1,8,9]$. In addition, several studies have demonstrated that the interaction between carotenoids and Chlorophylls play an important role as dissipation valves for excess excitation energy $[8,10,11]$. For example, in a study of Ruban et al. it has been proposed that conformational change upon aggregation gives rise to an increase of electronic interactions between lutein 1 and the surrounding chlorophylls, which result in an energy flow from chlorophylls to the first excited carotenoid state, $\mathrm{Car}_{1}$, of lutein 1 [9]. However, whether indeed a conformational change in antenna complexes is leading to the NPQ and if major or minor LHCs are involved is currently still controversially discussed $[14,15]$. A direct proof of electronic interactions between carotenoid $S_{1}$ states and chlorophylls in the 
energy dissipation is difficult. Especially, a quantitative analysis of the extent of these interactions and their direct involvement in the flow of excitation energy is not easy. One reason is that the electronic transition between the carotenoid ground state and its first excited state, Car $S_{1}$, is optically forbidden. This prevents a direct observation of the role of Car $S_{1}$ by conventional absorption or fluorescence spectroscopy. However, one convenient way to overcome this limitation is two-photon excitation (TPE) because the electronic transition between the carotenoid ground state and its first excited state is two-photon allowed [16-19]. Therefore, two-photon excitation is an advantageous tool which enables the selective excitation of carotenoids in LHC II and even in intact plants [2023]. The measurement of chlorophyll fluorescence upon two-photon excitation provides evidence for energy transfer from Car $S_{1}$ to Chl $\mathrm{Q}_{\mathrm{y}}$ state and can be used to evaluate the electronic interaction between Car $S_{1}$ and Chls. The coupling parameter $\left(\phi_{\text {Coupling }}^{\text {Car } S_{1}-\mathrm{Cl} l}\right)$, a ratio between the chlorophyll fluorescence observed upon two-photon excitation of the carotenoids, $\mathrm{F}^{\mathrm{TPE}}$, and the chlorophyll fluorescence observed upon direct chlorophylls one-photon excitation (OPE), $\mathrm{F}^{\mathrm{OPE}}$, is a quantitative measure for electronic interaction between the carotenoid dark state, Car $S_{1}$, and the lowest Chl states, $Q_{y}$. (for details see e.g. [1]):

$\phi_{\text {Coupling }}^{\text {Car } \mathrm{S}_{1}-\mathrm{Chl}} \propto \frac{\mathrm{Fl}^{\mathrm{TPE}}}{\mathrm{Fl}^{\mathrm{OPE}}}$

Recently, we have shown by this technique that the fluorescence quenching and photosynthetic down-regulation in LHC II and entire plants, qE, are very closely linked to electronic interactions between carotenoids and chlorophylls [1]. Based on this and observations such as correlated red-shifted fluorescence [24] and absorption spectra as well as simultaneous, instantaneous energy flow in the opposite direction $\left(\mathrm{Chl} \rightarrow \mathrm{Car}_{1}\right.$ ) $[1,9,25]$ we suggested that the formation of excitonic carotenoid-chlorophyll interactions is a major molecular, photophysical quenching mechanism. The formation of excitonic carotenoid-chlorophyll interactions provides a very effective dissipation valve for excess excitation energy [26]. When two pigments with similar excited state energies come close together, two new electronic states are formed, one has a lower energy level than the original pigment energies and the other has a higher energy level (Fig. 1). The excitonic state at the lower energy level can act as an energy trap for excess energy of the whole pigment pool. In addition, the two new electronic states mix at least partially the characteristics of the original pigments. The life time of Car $\mathrm{S}_{1}$ state is about $10-30$ ps [27] whereas the fluorescence life time of

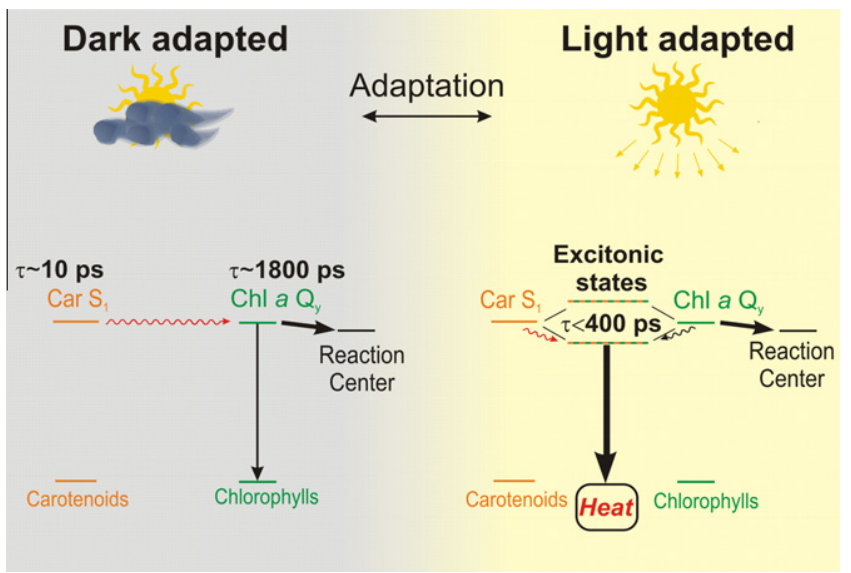

Fig. 1. Model of quenching excitonic interactions between carotenoids and chlorophylls [1]. Even small excitonic mixing of the short-living character of the first excited states of carotenoids, Car $\mathrm{S}_{1}$, with the long living Chl states will result in an effective trap and dissipation valve for excess excitation energy. the Chl a $\mathrm{Q}_{\mathrm{y}}$ state is about $1800 \mathrm{ps}$ [28]. Because the life time of Car $S_{1}$ state is much smaller than the fluorescence life time of the Chl a $\mathrm{Q}_{\mathrm{y}}$ state, even small excitonic mixing would cause drastic decreasing in chlorophyll fluorescence lifetime and consequently result in effective quenching of excess chlorophyll excitation energy by non-radiative deactivation.

In our previous study the quenching of LHC II was achieved by lowering the $\mathrm{pH}[1]$. However, the exact effect of this $\mathrm{pH}$ lowering on LHC II and its quenching was not $100 \%$ clear. The $\mathrm{pH}$ can either potentially induce conformational changes of single LHC II that causes the quenching and generation of electronic carotenoidchlorophyll interaction alone or lead to aggregation that either itself causes quenching or actually induces quenching conformational changes. It has been long known that isolated LHC II in surfactant solution is highly fluorescent, whereas for LHC II oligomers or crystals the fluorescence intensity decrease to several orders of magnitude $[8,14]$. It have been reported that the fluorescence quantum yield of aggregated LHC II is only $5 \%$ relative to non-aggregated LHC II [29]. For this reason the quenched LHC II has been thought to provide an in vitro model for the molecular mechanism for $\mathrm{qE}$ in vivo.

In the present study we performed a systematic investigation on the correlation between aggregation, quenching and electronic carotenoid-chlorophyll interactions of LHC II using two different methods, cation-induced precipitation and detergent removal with biobeads. For native LHC II the same linear correlation between $\phi_{\text {Coupling }}^{\mathrm{Car} \mathrm{S}_{1}-\mathrm{Chl}}$ and the fluorescence quenching can be observed as previously reported for the $\mathrm{pH}$ and Zea-dependent fluorescence quenching of LHC II [1]. In order to elucidate which chlorophyllcarotenoid pair might dominate the electronic interactions correlated to quenching we investigated in addition LHC II mutants lacking Chl 2 and Chl 13. These are chlorophylls that are neighboured to the carotenoids lutein 1 and neoxanthin, which both have been suggested to be the main quenchers in NPQ [8,9]. In addition, Chl 2 seems to possess one of the lowest chlorophyll excited state energies in LHC II due to excitonic strong interactions with Chl 1 and Chl $7[8,9,30,31]$ and is thus already a dominant receiver of excitation energy also under unquenched condition. However, we observed with these mutants again the same linear correlation as for native LHC II. This provides strong indication that these two chlorophylls play only a minor role for the observed effects. It is important to note, however, that this observation still does not exclude that their neighboured carotenoids lutein 1 and neoxanthin might form electronic interactions with other chlorophylls close by.

\section{Materials and methods}

The laser system has been described previously [1,20]. Briefly, a Vitesse Duo laser provided a $532 \mathrm{~nm}$ pump beam and $800 \mathrm{~nm}$ low energy femtosecond pulses. The repetition rate was $120 \mathrm{kHz}$. The femtosecond pulses were amplified by a mode-locked regenerative amplifier system (RegA 900) and used to pump an optical parametric amplifier (OPA 9450) for wavelength conversion. The idler beam tuned to $1188 \mathrm{~nm}$ was used for the two-photon excitation (TPE) of carotenoids. A hot mirror (L46-386, Edmund Optics) and a long pass filter $1100 \mathrm{~nm}$ (FEL 1100, Thorlabs) were used to thoroughly reject the visible light of the signal beam to prevent any undesired direct chlorophyll one-photon excitation. A confocal setup was used to focus the idler beam at the LHC II samples and collect the chlorophyll fluorescence. The fluorescence was detected by a photodiode (designed by Prof. D. Schwarzer) which was connected to the lock-in amplifier (EG\&G 5205, Dumberry, Canada). The lock-in amplifier was synchronized with a chopper which was put in the idler beam path. For one-photon excitation (OPE) 
a conventional PAM fluorometer (FMS1, Hansatech) was used. For the OPE measurement the modulating beam of $594 \mathrm{~nm}$ from the PAM fluorometer was used.

\subsection{Aggregated LHCII samples}

Native LHC II proteins were isolated from spinach as described in Ref. [27]. The aggregated LHC II was obtained by two different methods, cation-induced precipitation $[14,32,33]$ and detergent removal with biobeads [9,34]. With cation-induced precipitation LHC II concentrated stock was diluted 20 -fold with distilled water. $1 \mathrm{M}$ $\mathrm{MgCl}_{2}$ was added to reach the final concentration of $50 \mathrm{mM}$. The solution was homogeneously mixed and the precipitate was collected by centrifugation at $6000 \mathrm{rpm}$. Then the precipitate was washed two times with $50 \mathrm{mM} \mathrm{MgCl}$ before it was suspended in buffer solution (Tris pH $7.510 \mathrm{mM} \mathrm{MgCl}_{2}$ ). Surfactant DDM ( $n$ dodecyl- $\beta$-maltoside) was added to reach the final concentration $0.03 \%, 0.05 \%$, and $0.15 \%$ in order to obtain a range of aggregated LHC II. With detergent removal $2 \mathrm{ml}$ LHC II solution was incubated in a cuvette with $300 \mathrm{mg}$ SM-2 absorbent (Bio-Rad). The PAM fluorometer was used to measure the chlorophyll fluorescence during the incubation. A range of aggregated LHC II samples were obtained by sampling at different incubation times. The concentration of LHC II was adjusted to $\mathrm{OD}_{673 \mathrm{~nm}} 0.2-0.3 / \mathrm{mm}$ for the TPE and OPE measurement. Absorption and fluorescence spectra of LHC II were obtained by the UV/VIS spectrometer from Perkin-Elmer Lambda and the fluorescence spectrophotometer from Varian Cary Eclipse, respectively.

\subsection{LHC II mutants}

LHC II WT and mutants lacking certain chlorophyll were produced as described in Ref. [30]. In this experiment mutants lacking Chl $2(\Delta \mathrm{Chl} 2)$ and $\mathrm{Chl} 13(\Delta \mathrm{Chl} 13)$ were used. For the mutants detergent removal method with biobeads was used. Great care was taken to prevent protein removal from the solution.

\section{Results}

\subsection{Native LHC II}

In Fig. 2 it is shown that both LHC II aggregation methods - cation-induced precipitation and detergent removal with biobeads are successful to reduce the chlorophyll fluorescence of LHC II. The fluorescence quantum yield after direct one-photon excitation, $\mathrm{Fl}^{\mathrm{OPE}}$, reduces to $15 \%$ of the original value at $0.03 \%$ of DDM content.

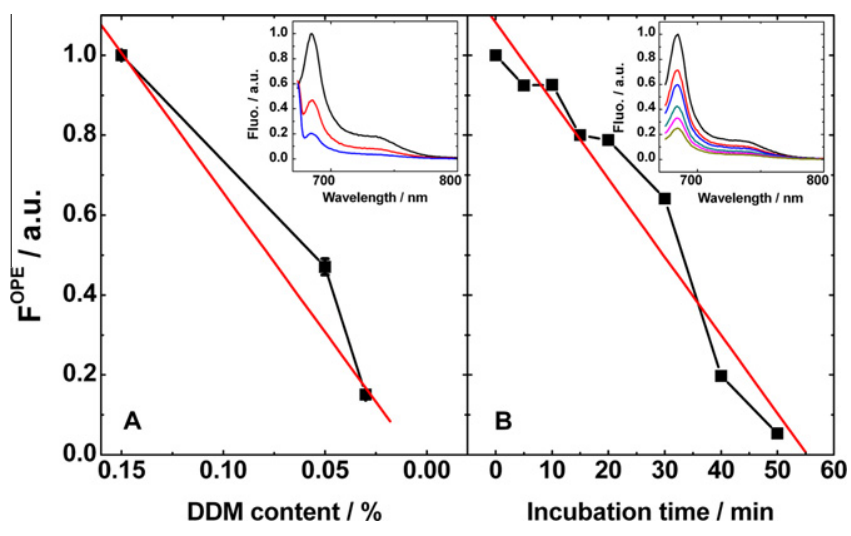

Fig. 2. Reduction of the chlorophyll fluorescence intensity of LHC II, $\mathrm{F}^{\mathrm{OPE}}$, by cationinduced precipitation (A) and detergent removal with biobeads (B). The insets show the corresponding fluorescence spectra.
With biobeads $\mathrm{Fl}^{\mathrm{OPE}}$ decreases even to $5 \%$ of the original value, when using incubation times of $50 \mathrm{~min}$. In the insets of Fig. 2 the fluorescence quenching of aggregated LHC II is also shown as decrease in the corresponding fluorescence emission spectra. It is worth noting that in the spectra of the LHC II solutions aggregated with cation-induced precipitation additional signals can be seen at shorter wavelengths which are not present in quenched LHC II samples prepared with biobeads. This is certainly due to the fact that the method of the cation-induced precipitation results in the formation of very large macro-aggregates which causes significant light scattering of the exciting beam at $673 \mathrm{~nm}$ [35]. That the formation of oligomers can be sufficient for extensive quenching in a similar manner as macro-aggregates has been previously reported [34,36,37].

In Fig. 3 absorption spectra of LHC II prepared with biobeads are shown along with the corresponding absorption difference spectra calculated by subtracting the spectrum of the unquenched LHC II from the spectra of the various different quenched LHC II samples. These spectra resemble the $\mathrm{pH}$-induced difference spectra reported in our previous study [1]. Intriguingly, the band at $\approx 681 \mathrm{~nm}$ shows the same linear relationship with the fluorescence quenching (Fig. 4) as we reported previously for the $\mathrm{pH}$ - and Zea-dependent quenching [1]. We calculated here again the NPQ parameter in the same way as in the study of Bode et al. by Eq. (2) and using the maximum fluorescence observed with the unquenched LHC II for the value of $F_{\mathrm{m}}$ and the quenched fluorescence intensities for the value of $F_{\mathrm{m}}^{\prime}$

$\mathrm{NPQ}=\frac{F_{\mathrm{m}}}{F_{\mathrm{m}}^{\prime}}-1$

The difference absorption spectra of LHC II prepared by the cationinduced precipitation show also significantly increased red-shifted bands (Fig. 5). However, around $650 \mathrm{~nm}$ negative peaks are observed and are most likely caused by the before mentioned scattering effects. An earlier study of Naqvi and co-workers explain these differences in the spectra of large macro-aggregates very convincingly in a theoretical approach by scattering and sieving effects [35]. Therefore, we will rely in the following for the quantification of the linear correlation between the red-shifted peak in the difference absorption spectra and the aggregation dependent fluorescence quenching only on the aggregation method of detergent removal with biobeads.

In Bode et al. we also showed that the coupling parameter, $\phi_{\text {Coupling }}^{\mathrm{Car} \mathrm{S}_{1}-\mathrm{Chl}}$, correlates linearly to NPQ in isolated LHC II and in vivo [1]. A very similar linear correlation was observed for plants during photosynthetic regulation (see inset in the lower left corner of

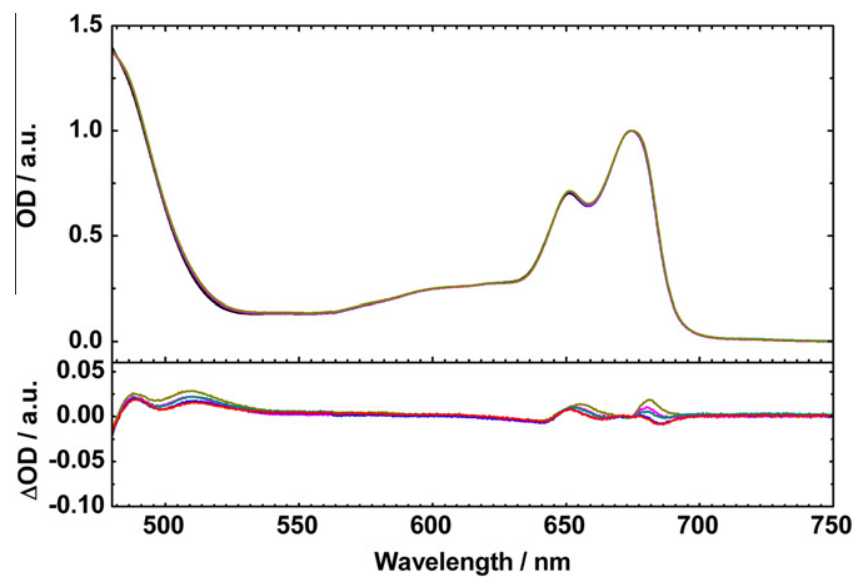

Fig. 3. Absorption spectra of LHC II incubated for different times with biobeads (top) along with the corresponding absorption difference spectra (bottom). 


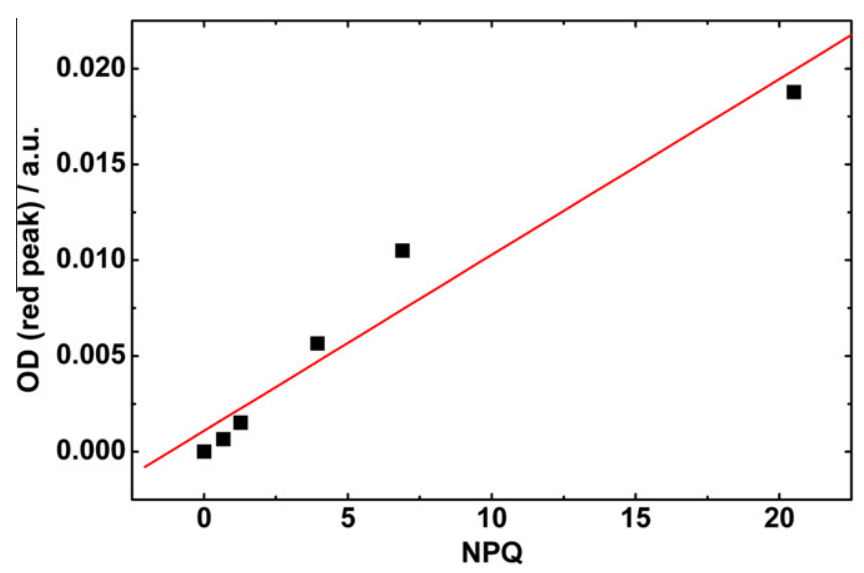

Fig. 4. Correlation between the red peak intensity in the absorption difference spectra at $681 \mathrm{~nm}$ in Fig. 3 and the fluorescence quenching, NPQ, for quenched LHC II prepared by detergent removal with biobeads. (For interpretation of the references to colour in this figure legend, the reader is referred to the web version of this article.)

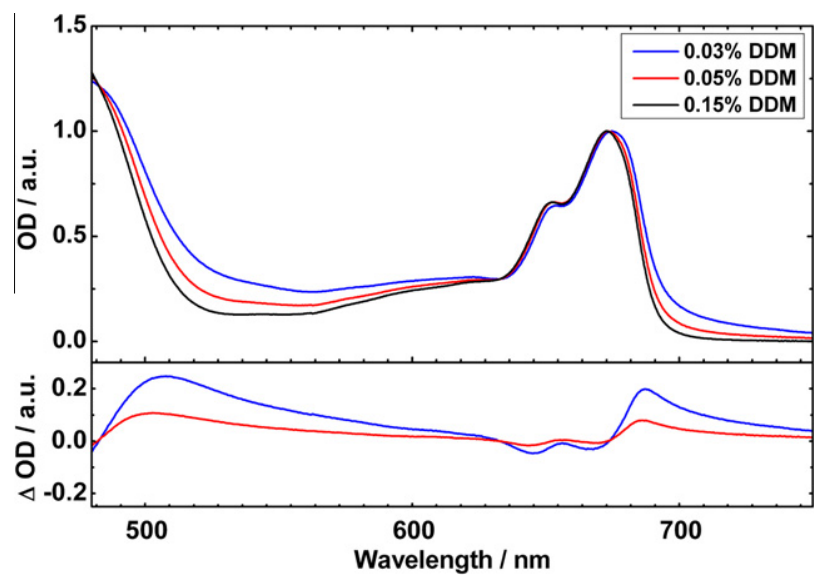

Fig. 5. Top: Absorption spectra of LHC II observed with different DDM contents (Fig. 1) along with the corresponding absorption difference spectra (bottom).

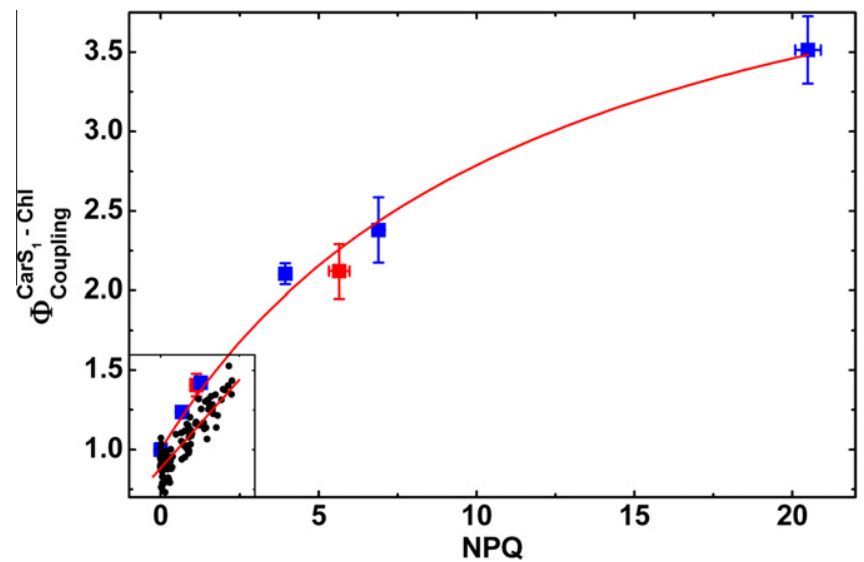

Fig. 6. Correlation of $\phi_{\text {Coupling }}^{\mathrm{Car} \mathrm{S}_{1}-\mathrm{Chl}}$ with the fluorescence quenching, NPQ, for quenched LHC II prepared by cation-induced precipitation (red squares) and detergent removal with biobeads (blue squares). The inset corresponds to the native regulation range and shows the in vivo data obtained for wild type Arabidopsis thaliana plants and various NPQ mutants (black circles) [1].

Fig. 6) as well as for $\mathrm{pH}$ - and Zea-dependent fluorescence quenching of LHC II. Therefore, we also determined here the correlation between these two parameters for the aggregation quenching of LHC II (Fig. 6). As can be seen in Fig. 6, very similar dependences between $\phi_{\text {Coupling }}^{\mathrm{Car} \mathrm{S}_{1} \mathrm{Chl}}$ and NPQ are obtained with the two different preparation methods. In addition they match very well the linear correlation observed for the pH-dependent quenching of LHC II and are therefore also very similar to the correlation observed in planta. The slope of the $\phi_{\text {Coupling }}^{\mathrm{Car} \mathrm{S}_{1}-\mathrm{Chl}} / \mathrm{NPQ}$ correlation of isolated LHC II is for small NPQ values about $20 \%$ higher than the in vivo correlation. However, whereas in the native regulation range as well as in the $\mathrm{pH}$-dependent LHC II quenching only NPQ parameters from 0 to $\sim 3$ can be observed, the aggregation of LHC II allows achieving fluorescence quenching far beyond that range corresponding to values of NPQ > 20. In this unnatural high quenching range a deviation from the linear correlation can be observed that is likely due to reabsorption effects or other non-linear saturation effects.

\subsection{LHC II mutants}

In order to elucidate which chlorophyll-carotenoid pair might dominate the observed correlations between $\phi_{\text {Coupling }}^{\mathrm{Car} \mathrm{S}_{1} \mathrm{Chl}}$ and NPQ, LHC II mutants lacking certain chlorophyll molecules were investigated. We investigated here two mutants that specifically lack Chl 2 and Chl 13. It is shown that the $\pi$ systems of Chl 2 and Lut 1 are exactly coplanar with an interplanar distance of $3.5 \AA$ and hence result in pronounced overlap of $\pi$ orbitals [38]. Furthermore, it has been reported that a conformational change induced by the changes in crystal packing would bring Lut 1 closer to Chl 2 [39]. In contrast, Chl 13 is a chlorophyll b molecule which has a higher energy level and normally is not considered as a quenching site. However, Chl 13 and $\mathrm{Chl}$ 10, constitute a special associated chlorophyll b dimer with the closest distance $3.5 \AA$ [8] and are close to neoxanthin which might have strong electronic interactions with chlorophylls [40]. We prepared aggregates of these two mutants with biobeads in the same way as the WT aggregates. We again measured the chlorophyll fluorescence upon one-photon and two-photon excitation to determine the parameters of $\phi_{\text {Coupling }}^{\mathrm{Car} \mathrm{S}_{1} \mathrm{Chl}}$ and NPQ. The aggregated WT and mutants showed the same incubation time dependent quenching-characteristics as native LHC II (data not shown). However, as shown in Fig. 7 no major difference in linear correlation between $\phi_{\text {Coupling }}^{\text {Car } S_{1}-\mathrm{Chl}}$ and NPQ was observed for these two mutants in comparison with the WT data. This provides

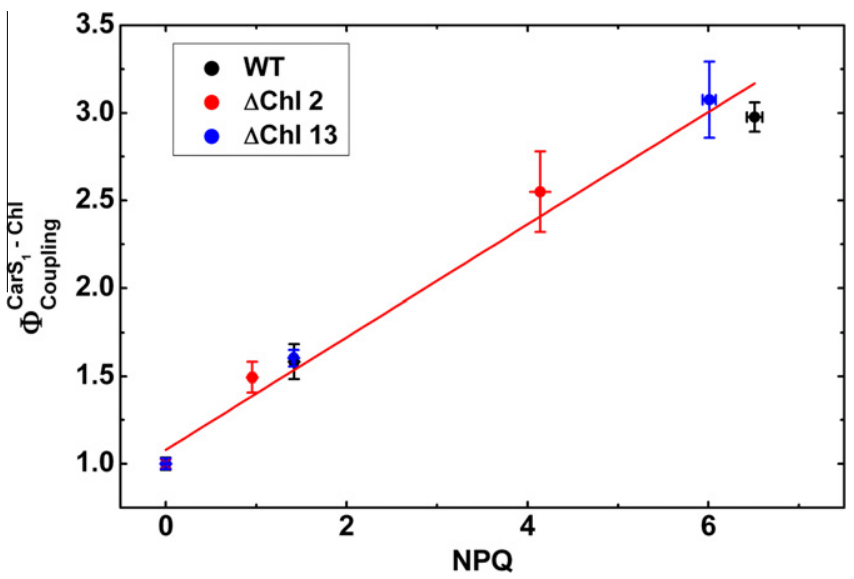

Fig. 7. Correlation between the red peak intensity in the absorption difference spectra at 681 and the fluorescence quenching, NPQ, for native LHC II (black) as well the mutants $\Delta \mathrm{Chl} 2$ (red) and $\Delta \mathrm{Chl} 13$ (blue). As in Fig. 4 the quenching was here achieved by detergent removal with biobeads. (For interpretation of the references to colour in this figure legend, the reader is referred to the web version of this article.) 
strong indication that these two chlorophylls play only a minor role for the observed carotenoid-chlorophyll interactions.

\section{Discussion}

In our previous study [1] the fluorescence quenching of isolated LHC II has been initiated by acidification and was enhanced by Zea. A linear correlation between the extent in the electronic coupling between carotenoids and chlorophylls, $\phi_{\text {Coupling }}^{\mathrm{Car} \mathrm{S}_{1} \mathrm{Chl}}$, and the extent in the fluorescence quenching has been observed. We attributed the linear correlation to the formation of excitonic states between carotenoids and chlorophylls resulting in low lying states that act as an energy trap and quench excess excitation energy. However, the exact effect of this pH lowering on LHC II and its quenching was not clear. We therefore present here a systematic study investigating the aggregation, quenching and electronic interactions between carotenoids and chlorophylls of LHC II using two different methods, cation-induced precipitation and detergent removal with biobeads. It turns out that both methods result in a very similar correlation between $\phi_{\text {Coupling }}^{\mathrm{Car} \mathrm{S}_{1}-\mathrm{Chl}}$ and the extend in the fluorescence quenching (Fig. 6) and that this correlation matches very well the linear correlation found for the $\mathrm{pH}$ - and Zea-dependent LHC II quenching [1]. This result clearly supports the assumption that the $\mathrm{pH}$-dependent LHC II quenching reported in our previous work is based on the same molecular mechanism as in LHC II aggregates.

Also, the same red-shifted absorption bands that have been previously observed for quenched LHC II at different $\mathrm{pH}$ values and Zea contents can be observed in the difference spectra of LHC II samples aggregated by detergent removal with biobeads (Fig. 3). Their intensity again correlates well and linearly with the extent in fluorescence quenching (Fig. 4). This is again supporting a model in which low lying carotenoid-chlorophyll excitonic states act as energy traps and dissipation valves for excess excitation energy (Fig. 1). The difference absorption spectra of LHC II aggregated by cation-induced precipitation look quite different (Fig. 5) but this can be easily explained by the formation of very large macroaggregates and resulting scattering effects and distortions of the absorptions spectra. This phenomenon has been described in detail in [35]. The fact that the difference absorption spectra of LHC II samples quenched by different $\mathrm{pH}$ values and Zea contents looks similar to difference absorption spectra of LHC II samples aggregated by detergent removal with biobeads provides indication that acidification of LHC II induces the formation of smaller oligomers. However, whether this oligomerization directly leads to new electronic interactions of carotenoids and chlorophylls at the periphery of LHC II or is first resulting in conformational changes that induces changing electronic interactions within LHC II cannot be finally answered by this finding.

It might be surprising that a forbidden state can participate in excitonic interactions. However, it is long known that it is also possible that an effective energy transfer, Car $\mathrm{S}_{1} \rightarrow$ Chl, can occur between carotenoid dark states and nearby chlorophylls [17,27]. This becomes possible when the pigments are in very close contact so that local interactions of the electronic wave functions become important [41]. Since there is no substantial difference in the electronic couplings that enables energy transfer and the electronic couplings that cause excitonic interactions the experimental finding that effective Car $\mathrm{S}_{1} \rightarrow$ Chl energy transfer is possible provides evidence that also excitonic states should be formed between Car $\mathrm{S}_{1}$ and Chl states when the pigments are oriented in the right way and have similar state energies. However, whether an orientation change between the pigments or an energy shift of the pigments is the cause of the enhancement of such interactions in LHC II and what is actually inducing this remains an open question and also needs further investigations.
In order to further explore the exact molecular site which dominants the observed linear correlations, we explored in the present study also the chlorophyll deficient LHC II mutants $\Delta$ Chl 2 and $\Delta$ Chl 13. In different studies Chl 2 and Chl 13 have been proposed to couple with Lut 1 and Neo, respectively, as possible quenching sites in LHC II $[8,9]$. But again, WT LHC II and these two mutants show the same linear correlation between the coupling parameter, $\phi_{\text {Coupling }}^{\text {Car S }_{1} \text {, }}$, and NPQ (Fig. 7). This rather indicates that neither Chl 2 nor Chl 13 cause the specific electronic or excitonic interactions that correlate with the quenching. However, that definitively does not rule out that the carotenoids, Lut 1 and $\mathrm{Neo}$, participate in the quenching mechanism. They well might form electronic interactions with other neighboured chlorophylls than Chl 2 or Chl 13. Also, the mutants might be distorted due to the fact that specific chloropohylls and also carotenoids are missing. For example, the analytical data of HPLC show that the $\triangle \mathrm{Chl} 13$ mutant contains only trace amounts of Vio and Neo. Therefore, to elucidate which chlorophylls and carotenoids are exactly involved in the observed correlation further investigations with other LHC II mutants are necessary and will be subject of future studies.

Our observations also support the assumption that Zea rather seem to facilitate the transformation of light harvesting complexes into the quenched state but is not necessarily involved in the quenching itself. In vivo, the rapidly reversible energy-dependent quenching ( $\mathrm{qE}$ ) requires the build-up of trans-thylakoid $\mathrm{pH}$ gradient $(\Delta \mathrm{pH})$, which triggers the enzymatic conversion of xanthophyll-cycle carotenoid violaxanthin (Vio) to zeaxanthin. Two explanations about the role of Zea in the fluorescence quenching have been proposed. One hypothesis is that the first excited state of Zea directly acts as a quencher, while Vio is not capable of quenching $[20,21]$. The other is that Vio and Zea regulates $\mathrm{qE}$ in an allosteric model in which Zea promotes the formation of quenching states while Vio cannot [42]. For example, it has been shown that the fluorescence quenching of isolated LHC II in high detergent concentration can be facilitated by the addition of activators, zeaxanthin and auroxanthin [43] or by lowering the $\mathrm{pH}$ [44]. In the present study, none of the investigated samples contained Zea but still the same correlation between $\phi_{\text {Coupling }}^{\mathrm{Car} \mathrm{S}_{1}-\mathrm{Chl}}$ and the very effective quenching can be observed (Fig. 6). This observation supports the idea that Zea facilitates the transformation of light harvesting complexes into quenched states but is not necessarily involved in the quenching itself. However, it is not excluded that Zea is actually the active quencher but can in its absence be replaced by other carotenoids. Also, such conclusion depend on the question whether aggregated, quenched LHC II indeed provides a good in vitro model for the molecular mechanism for $\mathrm{qE}$ in vivo. At least the fact that the slope of the correlation between $\phi_{\text {Coupling }}^{\text {Car } \mathrm{S}_{1}-\mathrm{Chl}}$ and the fluorescence quenching of in vivo and in vitro measurements are very similar is supportive for similar quenching mechanism in both cases (Fig. 6, inset).

In contrast to our previous studies on the correlation between $\phi_{\text {Coupling }}^{\mathrm{Car} \mathrm{S}_{1}-\mathrm{Chl}}$ and the extend in the fluorescence quenching the aggregation of LHC II samples allows to achieve an extend in quenching that is far beyond the native regulation range of NPQ up to at most $\sim 3$. We here observe that higher quenching - corresponding to NPQ values of $>5$ - results in increasing deviations with increasing quenching from the previously reported linear dependence between $\phi_{\text {Coupling }}^{\text {Car } S_{1}-\text { anl }}$ and NPQ (Fig. 6). Given the extreme quenching that corresponds to NPQ values of $>20$ this is probably not surprising but further studies are necessary to elucidate if this is simply due to effects like reabsorption or are indicative for further details on the molecular quenching mechanism.

As previously mentioned, the data of all plant variants, wild type and mutants, which cover a wide rage of NPQ capacities, show a very similar linear correlation between the coupling parameter, $\phi_{\text {Coupling }}^{\text {Car } \mathrm{S}_{1} \text { Chl }}$, and NPQ as observed in the $\mathrm{pH}-$ as well as the aggrega- 
tion dependent quenching of LHC II (Fig. 6, inset). The value of $\phi_{\text {Coupling }}^{\text {Car } S_{1}-\mathrm{Chl}}$ in vivo is about $20 \%$ less than in isolated LHC II. This can certainly be attributed to the fact, that in planta not only LHC II is observed. Even though LHC II is also the dominating pigment-protein complex in vivo, the method determines $\phi_{\text {Coupling }}^{\mathrm{Car} \mathrm{S}_{1}-\mathrm{Ch}}$ for all pigment-protein complexes of the entire photosynthetic apparatus simultaneously. This is still true when the detected fluorescence originates in plant measurements only from a few distinct chlorophylls: the measured values of $\phi_{\text {Coupling }}^{\mathrm{Car} \mathrm{S}_{1} \text {-Chl }}$ represent an average of the electronic interactions of all carotenoid-chlorophyll pairs. However, we believe the small deviation of only $20 \%$ in the slope between the in vivo and the in vitro measurements is an indication that also in planta the observed results are dominated by interactions in LHC II. Certainly it is also possible that other pigment-protein contribute to a larger extend to the correlation observed in plants but have a similar quenching dependence between $\phi_{\text {Coupling }}^{\mathrm{Car} \mathrm{S}_{1} \mathrm{Chl}}$ and the quenching as LHC II.

\section{Conclusions}

We have shown in the present study that the linear correlation between the extend in the electronic coupling between carotenoids and chlorophylls, $\phi_{\text {Coupling }}^{\mathrm{Car} \mathrm{S}_{1} \text {-Chl }}$ and the fluorescence quenching ("NPQ") induced in LHC II by cation-induced precipitation or detergent removal with biobeads is the same as the previously reported $\mathrm{pH}$ - and Zea-dependent correlation of native LHC II [1] (Fig. 6, inset). In addition, the same linear correlation between red-shifted absorption bands and the fluorescence quenching for LHC II aggregated by detergent removal with biobeads is observed (Fig. 4). This is supporting a model in which low lying carotenoid-chlorophyll excitonic states act as energy traps and dissipation valves for excess excitation energy (Fig. 1) and provides indication that the $\mathrm{pH}-$ and zeaxanthin induced quenching of LHC II is based on the formation of small oligomers similar to the quenching induced by detergent removal with biobeads.

In order to elucidate which chlorophyll-carotenoid pair might dominate this correlation we also investigated the LHC II mutants $\Delta \mathrm{Chl} 2$ and $\Delta \mathrm{Chl} 13$ lacking the corresponding chlorophyll molecules. However, the fact that the same linear correlation was also observed for these two mutants (Fig. 7) provides indication that these two chlorophylls play only a minor role for the observed effects. It is nevertheless important to note that our results do not exclude that carotenoids lutein 1 and neoxanthin neighboured to Chl 2 and Chl 13 might be involved in the formation of quenching carotenoid-chlorophyll interaction. For example, they might interact with other chlorophylls than Chl 2 and Chl 13. Therefore, further studies with other LHC II mutants are necessary to elucidate which chlorophylls and carotenoids are exactly involved in the observed correlation between quenching and electronic interactions.

\section{Acknowledgements}

We thank W. Kühlbrandt for his kind collaboration. This work was supported by the Fonds der Chemischen Industrie (DFG), the Deutsche Forschungsgemeinschaft.

\section{References}

[1] S. Bode, C.C. Quentmeier, P.-N. Liao, N. Hafi, T. Barros, L. Wilk, F. Bittner, P.J. Walla, Proc. Natl. Acad. Sci. USA 106 (2009) 12311.

[2] B. Demmig-Adams, W.W. Adams, Science 298 (2002) 2149.

[3] C. Kühlheim, S. Agren, S. Jansson, Science 297 (2002) 91.

[4] P. Horton, A.V. Ruban, R.G. Walters, Ann. Rev. Plant Physiol. Plant Mol. Biol. 47 (1996) 655.

[5] P. Müller, X.-P. Li, K.K. Niyogi, Plant Physiol. 125 (2001) 1558.

[6] G.H. Krause, E. Weis, Ann. Rev. Plant Physiol. Mol. Biol. 42 (1991) 313.

[7] X.P. Li, O. Björkman, C. Shih, A.R. Grossman, M. Rosenquist, S. Jansson, K.K. Niyogi, Nature 403 (2000) 391.

[8] A.A. Pascal, Z. Liu, K. Broess, B.V. Oort, H.V. Amerongen, C. Wang, P. Horton, B. Robert, W. Chang, A. Ruban, Nature 436 (2005) 134.

[9] A.V. Ruban, R. Berera, C. Ilioaia, I.H.M. Van Stokkum, J.T.M. Kennis, A.A. Pascal, H. Van Amerongen, P. Horton, R. Van Grondelle, Nature 450 (2007) 575.

[10] H.A. Frank, J.A. Bautista, J.S. Josue, A.J. Young, Biochemistry 39 (2000) 2831.

[11] N.E. Holt, D. Zigmantas, L. Valkunas, X.-P. Li, K.K. Niyogi, G.R. Fleming, Science 307 (2005) 433.

[12] T.K. Ahn, T.J. Avenson, M. Ballottari, Y.-C. Cheng, K.K. Niyogi, R. Bassi, G.R. Fleming, Science 320 (2008) 794.

[13] S. Amarie, J. Standfuss, T. Barros, W. Kühlbrandt, A. Dreuw, J. Wachtveitl, J. Phys. Chem. B 111 (2007) 3481.

[14] T. Barros, A. Royant, J. Standfuss, A. Dreuw, W. Kühlbrandt, EMBO J. 28 (2009) 298.

[15] T.J. Avenson, T.K. Ahn, D. Zigmantas, K.K. Niyogi, Z. Li, M. Ballottari, R. Bassi, G.R. Fleming, J. Biol. Chem. 283 (2008) 3550.

[16] S. Shima, R.P. Ilagan, N. Gillespie, B.J. Sommer, R.G. Hiller, F.P. Sharples, H.A. Frank, R.R. Birge, J. Phys. Chem. A 107 (2003) 8052.

[17] P.J. Walla, P.A. Linden, C.P. Hsu, G.D. Scholes, G.R. Fleming, Proc. Natl. Acad. Sci. USA 97 (2000) 10808.

[18] P.J. Walla, J. Yom, B.P. Krueger, G.R. Fleming, J. Phys. Chem. B 104 (2000) 4799 .

[19] A.P. Shreve, J.K. Trautman, T.G. Owens, A.C. Albrecht, Chem. Phys. Lett. 170 (1990) 51.

[20] S. Bode, C.C. Quentmeier, P.-N. Liao, T. Barros, P.J. Walla, Chem. Phys. Lett. 450 (2007) 379.

[21] A. Wehling, P.J. Walla, Photosynth. Res. 90 (2006) 101.

[22] A. Wehling, P.J. Walla, J. Phys. Chem. B 109 (2005) 24510.

[23] M. Hilbert, A. Wehling, E. Schlodder, P.J. Walla, J. Phys. Chem. B 108 (2004) 13022.

[24] M.P. Johnson, A.V. Ruban, J. Biol. Chem. 284 (2009) 23592.

[25] Y.Z. Ma, N.E. Holt, X.P. Li, K.K. Niyogi, G.R. Fleming, Proc. Natl. Acad. Sci. USA 100 (2003) 4377.

[26] H. Van Amerongen, R. Van Grondelle, J. Phys. Chem. B 1005 (2001) 604

[27] T. Polívka, V. Sundström, Chem. Rev. 104 (2004) 2021.

[28] A.M. Gilmore, T.L. Hazlett, Proc. Natl. Acad. Sci. USA 92 (1995) 2273.

[29] B. Van Oort, A. Van Hoek, A.V. Ruban, H. Van Amerongen, FEBS Lett. 581 (2007) 3528.

[30] R. Van Grondelle, V.I. Novoderezhkin, Phys. Chem. Chem. Phys. 8 (2006) 793.

[31] J.S. Frähmcke, P.J. Walla, Chem. Phys. Lett. 430 (2006) 397.

[32] Z.H. Hu, F. Zhou, C.H. Yang, Photosynthetica 44 (2006) 615.

[33] J.J. Burke, C.L. Ditto, C.J. Arntzen, Arch. Biochem. Biophys. 187 (1978) 252.

[34] C. Ilioaia, M.P. Johnson, P. Horton, A.V. Ruban, J. Biol. Chem. 283 (2008) 29505

[35] K.R. Naqvi, T.B. Melø, B.B. Raju, T. Jávorfi, G. Garab, Spectrochim. Acta Part A 53 (1997) 1925.

[36] P. Zuo, H. Chen, Y. Wu, S. Shen, P. Wang, X. Al, J. Zhang, L. Li, T. Kuang, Chin. Sci. Bull. 51 (2006) 1444.

[37] A.V. Ruban, P. Horton, Biochim. Biophys. Acta 1102 (1992) 30.

[38] J. Standfuss, A.C.T. Van Scheltinga, M. Lamborghini, W. Kühlbrandt, EMBO J. 24 (2005) 919.

[39] H. Yan, P. Zhang, C. Wang, Z. Liu, W. Chang, Biochem. Biophys. Res. Commun. 355 (2007) 457.

[40] R. Croce, R. Remelli, C. Varotto, J. Breton, R. Bassi, FEBS Lett. 456 (1999) 1.

[41] C.-P. Hsu, P.J. Walla, M. Head-Gordon, G.R. Fleming, J. Phys. Chem. B 105 (2001) 11016.

[42] P. Horton, M.P. Johnson, M.L. Perez-Bueno, A.Z. Kiss, A.V. Ruban, FEBS Lett. 275 (2008) 1069

[43] M. Wentworth, A.V. Ruban, P. Horton, FEBS Lett. 471 (2000) 71

[44] M. Wentworth, A.V. Ruban, P. Horton, Biochemistry 40 (2001) 9902. 